Europhys. Lett., 69 (5), pp. 739-745 (2005)

DOI: $10.1209 /$ epl/i2004-10408-x

\title{
Order and disorder in columnar joints
}

\author{
L. Goehring and S. W. Morris \\ Department of Physics, University of Toronto - 60 St. George Street \\ Toronto, Ontario, M5S 1A7, Canada
}

received 23 June 2004; accepted in final form 23 December 2004

published online 2 February 2005

PACS. 45.70.Qj - Pattern formation.

PACS. 62.20.Mk - Fatigue, brittleness, fracture, and cracks.

\begin{abstract}
Columnar joints are three-dimensional fracture networks that form in cooling basalt and several other media. The network organizes itself into ordered, mostly hexagonal columns. The same pattern can be observed on a smaller scale in desiccating starch. We show how surface boundary conditions in the desiccation of starch affect the formation of columnar joints. Under constant drying power conditions, we find a power law dependence of columnar cross-sectional area with depth, while under constant drying rate conditions this coarsening is eventually halted. Discontinuous transitions in pattern scale can be observed under constant external conditions, which may prompt a reinterpretation of similar transitions found in basalt. Starch patterns are statistically similar to those found in basalt, suggesting that mature columnar jointing patterns contain inherent residual disorder, but are statistically scale invariant.
\end{abstract}

Columnar jointing, in which shrinkage fractures arrange themselves to leave behind a pattern of mainly hexagonal pillars, has fascinated scientists and naturalists for centuries [1-3]. The Giant's Causeway in Northern Ireland and the Devil's Postpile in California are famous examples. Columnar joints occur in lava flows, sandstone, mud, coal, glass, starch, and ice [3-11], while a $2 \mathrm{D}$ variant may cause ice/sand-wedge polygons in permafrost on Earth and Mars [5,12]. Columnar joints are formed through the self-organization of shrinkage cracks in a three-dimensional material, as either heat or moisture is removed from one surface $[6,13,14]$. Brittle fracture is initiated by the stress buildup at this surface [14], and the resulting cracks progressively extend, tracking a shrinkage front as it moves into the bulk $[15,16]$. At any given time, active fractures are confined to a nearly planar layer between the remaining compliant material, and the fully fractured material $[15,16]$. The evolution of this quasi-2D polygonal pattern through time is recorded in the depth dependence of the resulting prismatic columns. Often, in geophysical examples, this pattern only becomes apparent when the interior of the formation is exposed by erosion and weathering. As a result, previous geophysical studies of the fracture pattern have been limited to exposed planar surfaces. Columnar jointing is a surprisingly general phenomenon, well known in igneous rocks (both terrestrial [13] and lunar [17]), but also seen in sedimentary [5,10], and metamorphic rocks [5], as well as in manmade and biological materials [4-9]. Examples in basalt and corn starch are shown in fig. 1. Joints can range in size from micron sized diffusively cooled columns in vitrified, impure, ice [11] to meter sized evaporatively cooled columns in basalt [14].

(C) EDP Sciences 

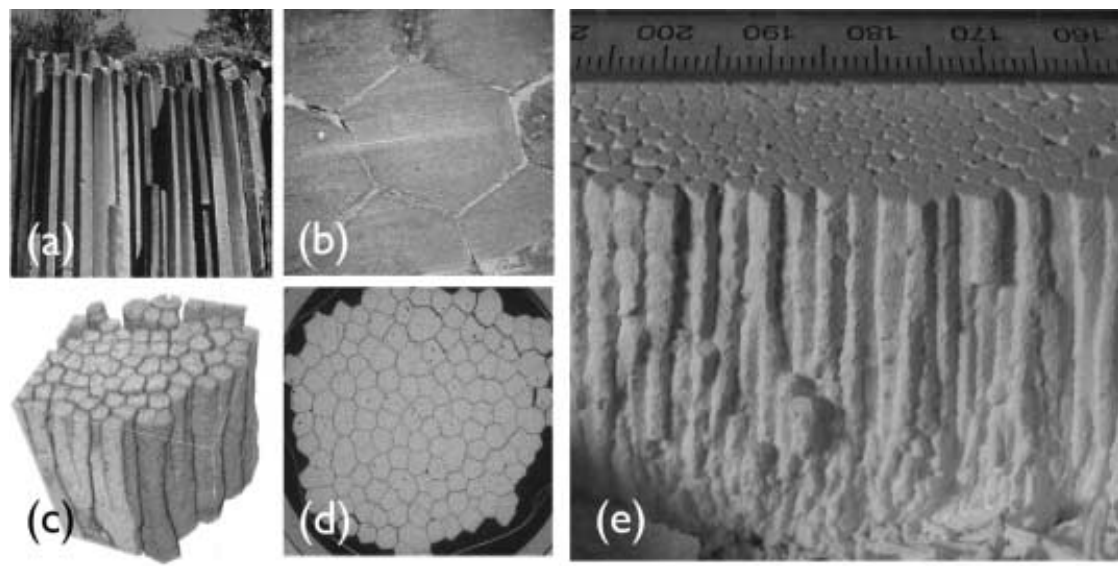

Fig. 1 - Examples of columnar jointing. (a) The colonnade of the Devil's Postpile, CA, USA. (b) Exposed surface of the Devil's Postpile, showing the quasihexagonal fracture pattern, as it occurs in basalt. (c) MicroCT X-ray tomography image of corn starch colonnade with $36 \mu \mathrm{m}^{3}$ voxel resolution, and $2.5 \mathrm{~cm} /$ side. (d) Cross-section of a tomogram at a depth of $18 \mathrm{~mm}$. (e) A typical corn starch colonnade (shown inverted) studied in these experiments, grown with a constant evaporation rate.

Other polygonal fracture patterns confined to a plane may evolve and order by similar processes, if a mechanism for the lateral motion of cracks exists. For example, fractured freeze-thaw polygons, with mobile edges, are observed in Arctic and Antarctic permafrost $[18,19]$. Similar features have been imaged on Mars, suggesting the presence of Martian permafrost $[19,20]$. Recent phase field models of columnar jointing have been constructed to apply equally to all these cases [12]. This generality suggests that a detailed description of columnar jointing, based on controlled, repeatable experiments, could have applicability to a number of fields.

To date, due to the constraints on geophysical observation, there exists no quantitative description of how columnar joints order. In the absence of such experimental or field data, a number of theories have been proposed [12,13,21-23]. However, there is no consensus on exactly how or why these joints order. Nor is it understood how surface boundary conditions and material properties contribute to the columnar structure and scale. In this paper, we present the first ever $3 \mathrm{D}$ data describing columnar jointing, specifically describing the ordering and coarsening processes observed near the free surface.

Our experimental techniques are based on those of Müller [6-8], who independently rediscovered the jointing in starches previously noted by Huxley [3] and by French [4]. These techniques have also recently been adopted by Toramaru and Matsumoto [9], who investigated the relationship between desiccation rate and the starch pattern at a fixed depth.

$250 \mathrm{~W}$ heat lamps were used to dry 1: 1 slurries of 100\% pure corn starch and water. Traces of bleach were used to sterilize the experiment. We studied samples 1-100 mm deep, dried in circular flat-bottomed dishes; evaporation rates were between $10-40 \mathrm{mg} / \mathrm{h} \mathrm{cm}^{2}$, and samples typically dried between 1 and 28 days. Water content was measured by weighing the samples once per minute on an automated scale, as shown in fig. 2. Desiccation rates were set by regulating the heating and ventilation applied to the top surface of the starch. In one case overhead lamps at a fixed starch-lamp distance supplied a continuous, constant drying power. The drying power could be changed between experiments by varying the lamp-starch distance. In our second setup we used the measured sample weight, $m(t)$, to drive a feedback loop which 


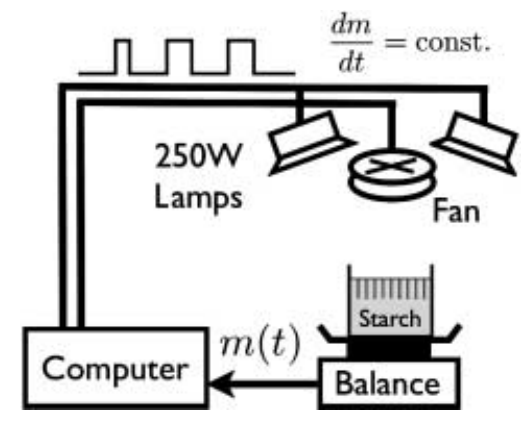

Fig. 2 - Experimental methods. An automated scale was used to feedback control the rate of evaporation. After drying, the fracture pattern is measured using a combination of MicroCT X-ray tomography and destructive sampling.

controlled the duty cycle of the heat lamps and a small fan. We used this feedback to keep the evaporation rate, $\mathrm{d} m / \mathrm{d} t$, constant. We shall refer to these methods as constant-power and constant-rate conditions, respectively. This level of control has not been available in the previous experiments of Müller [6-8] or Toramaru and Matsumoto [9].

As a starch slurry dries, first-generation cracks appear, penetrating through the entire sample depth and breaking the sample into large, disconnected polygons. Later, much finer secondary cracks initiate at the top surface, and propagate into the sample. The secondary crack front leaves behind columnar joints within each large polygon. This two-step fracture ensures that container size and shape do not affect the columnar-joint pattern.

We have used MicroCT X-ray tomography, shown in fig. 1c, d, to produce fully 3D visualizations of the pattern of columnar jointing in desiccating starch under several drying conditions. We have also recorded this pattern in cross-section by measuring the counterparts left on the drying containers after the starch is removed, and by sawing samples open in order to destructively observe the pattern at different depths. The violent nature of this destructive sampling constrains its use to samples $>1 \mathrm{~cm}$ height, limiting data collection in some cases.

Figure 3a shows the evolution of the average columnar cross-sectional area as a function of the depth. Under constant-power conditions, the evaporation rate drops steadily, as it becomes more difficult to drive water from the interior of the sample. The rate of change of evaporation suggests that water transport is partially driven by a wicking process, as is seen in the drying of other suspensions [16], and not, as Müller [6] assumed, entirely by diffusion. In fact, both diffusive heat transport and convection in the cracks are active in basalt [24]. In our starch, water transport is by wicking through the bulk of the material, rather than via the cracks. These conditions result in a slowly decelerating drying front, and a power law coarsening of the columnar area. This limited power law breaks down in several suggestive ways. The fracture scale is limited by a fine surface crack pattern, which is well fit by adding a small constant to the power law. This suggests that the mature pattern scale is independent of the surface fracture scale. In constant-rate experiments, after an initial transient, the drying front moves at constant speed, and the coarsening of the pattern is almost entirely halted. The transient coarsening is indistinguishable from that observed using constant external drying conditions.

In deep $(>5 \mathrm{~cm})$ samples, sudden transitions in scale were observed to occur (see fig. $3 \mathrm{~b}$ ). These transitions are sharp, quite reproducible, and separate colonnades of very different scales. Within the transition region, the fracture pattern shows increased disorder, as shown in fig. 3c. The inspection of tomograms shows that mergers of two or three columns, through 

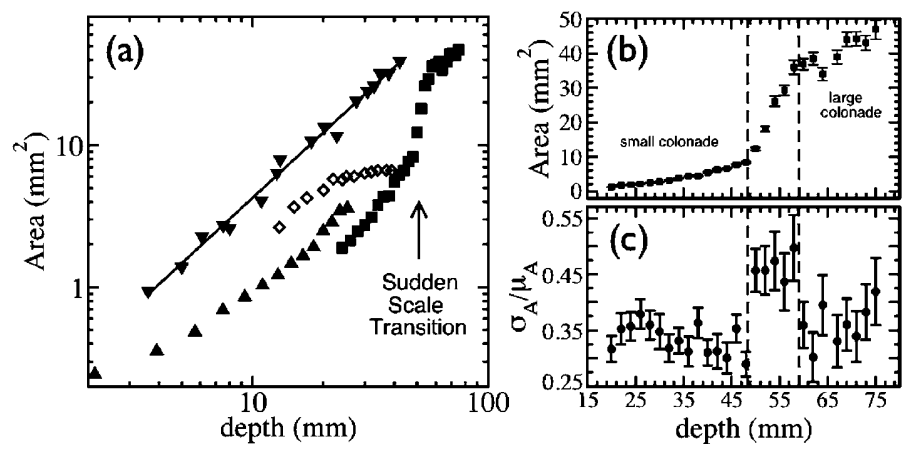

Fig. 3 - The coarsening behaviour of corn starch was studied in several drying situations. (a) Generally, for samples with constant power (solid symbols), a crude power law coarsening of cross-sectional area with depth was seen, with exponents of 1.6 to 2.2 (the line shown has a slope of 1.6). As expected [13], faster drying rates produce smaller columns. The columns shown with inverted triangles were grown with an initial drying rate of $\sim 10 \mathrm{mg} / \mathrm{h} \mathrm{cm}^{2}$, squares represent experiments with an initial drying rate of $\sim 32 \mathrm{mg} / \mathrm{h} \mathrm{cm}^{2}$. For samples dried with constant-rate conditions (open symbols), the coarsening is halted. In cases of deeper samples (squares), a sudden transition in scale was observed, without an accompanying discontinuity in the drying rate. (b) This transition (between dashed lines) is a sharp discontinuity in scale, and is associated with increased pattern disorder. (c) shows the relative disorder, as measured by the standard deviation of the area distribution $\left(\sigma_{A}\right)$, divided by the mean of the area distribution $\left(\mu_{A}\right)$.

the termination of a common fracture or junction, are the only events that can lead to pattern coarsening. Figure 4 shows several mechanisms of column evolution. New columns were occasionally created at existing vertices, but no columns were seen to vanish by constricting into a vertex. These observations imply that the transition regions are zones of greatly enhanced merger rate. This naturally increases the variance of the average column area during the transition.

Pattern disorder is increased during a sudden scale transition, but some ordering process acts efficiently to return the value of the relative variation in area to its pre-transition value of 0.35 within one data point $(2 \mathrm{~mm})$ of the end of such a transition. Similar ordering occurs near the drying surface. We investigated this ordering behaviour in a $28 \mathrm{~mm}$ deep, continually coarsening, starch colonnade, to study how a disordered surface crack network matured. The plots in fig. 5 show the depth dependence of four statistics we used to quantify disorder.

We observe the evolution of an initially disordered surface fracture pattern into a wellordered colonnade. To quantify this ordering, we have studied the distributions of areas, joint angles, and the number of neighbours as a function of depth, measured from the drying surface after complete desiccation, in cross-sections of the tomogram. Note that the number of neighbours, unlike the number of sides, which is traditionally reported, must average 6 for a polygonal tiling of the plane that avoids X-junctions [25].

All four starch statistics reach a statistically steady state after $\sim 1 \mathrm{~cm}$ of evolution, maintaining fixed, but large values thereafter. This indicates that considerable disorder remains in the pattern. Furthermore, in all four cases, the plateau values of the starch statistics match the values for the Giant's Causeway [2], a mature, well-ordered basalt pattern [13]. In this experiment, it could be argued that the residual disorder is dictated by the continued coarsening. However, we observe statistically similar descriptions of the limiting pattern in both the constant-rate experiments, for which coarsening is effectively halted, and the exposed features 

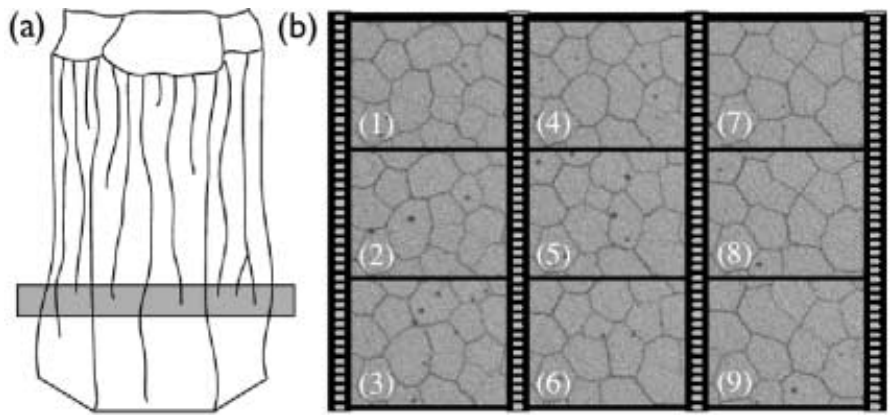

Fig. 4 - Pattern coarsening processes. (a) sketches the essential features of coarsening through fracture termination, the motility of the crack edges, and column creation from existing vertices. The upper surface is covered with a fine network of fractures, which we have omitted for simplicity. The grey region demonstrates where a burst of crack termination causes a sudden change in pattern scale. (b1-9) show the pattern features in real space, and are selected from a tomogram volume-filling image. Each panel shows a $4.5 \times 3.5 \mathrm{~mm}^{2}$ cross-section from approximately the centre of the volume, at depths increasing by $360 \mu \mathrm{m}$ per panel, beginning at a depth of $10.0 \mathrm{~mm}$. The black circles are bubbles, and the faint stripes are tomographic artifacts. Note how mobile the column edges can be. Column merger occurs between two pairs of columns near the centre of panel 1, and between panels 3 and 4 , in the lower right corner. Either a single crack, or a vertex can cease to propagate, and the merged column rapidly readjusts its shape and cross-sectional area to match its surroundings. Between panels 3 and 4, a new column appears from a vertex near the centre of the image.

of the non-coarsening basalt of the Giant's Causeway. The similarity in statistics from two very different systems, desiccated starch and cooled basalt, combined with evidence of a strong ordering process away from the common limiting pattern, suggests that residual disorder is intrinsic to the quasihexagonal pattern of columnar jointing. This is contrary to the frequently
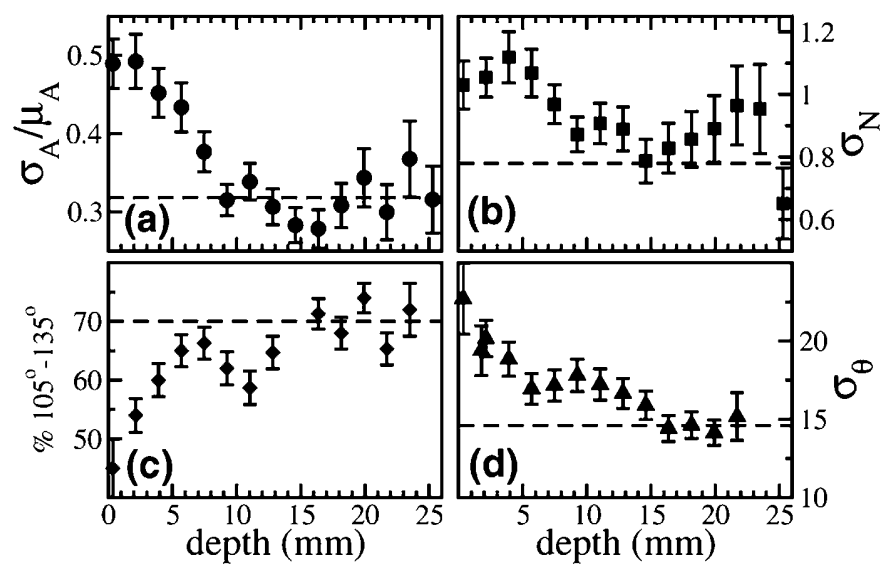

Fig. 5 - Statistical comparison of a tomography study of a $28 \mathrm{~mm}$ deep corn starch sample dried with constant power (data points, corresponding with data shown in upright triangles in fig. 3a), and O'Reilly's 1879 survey of the Giant's Causeway [2] (dashed lines). (a) The relative variation in column cross-sectional area. (b) Standard deviation of the distribution of number of neighbours $\left(\sigma_{N}\right)$. (c) $\%$ of Y-joints, here defined as those within $15^{\circ}$ of a $120^{\circ}$ joint. (d) Standard deviation in the distribution of joint angles $\left(\sigma_{\theta}\right)$, in degrees. 
encountered assumption that columnar jointing tends towards a perfect hexagonal lattice in cross-section [13,21]. However, such an assumption is based on the argument that hexagonal fracture maximizes the elastic energy release [21]. In strongly non-equilibrium situations, such as this one, energy arguments are not necessarily valid.

Our results can be used to explain a puzzling feature of basaltic columnar joints. A single basalt flow can contain several adjacent colonnades, with different scales, separated by disordered zones (entablature) often less than $1 \mathrm{~m}$ wide [26, 27]. Some colonnade-colonnade boundaries occur when cooling fronts travelling from the top and bottom of a flow collide. However, other transitions remain unexplained, except through proposed catastrophic events such as intermittent flooding [27,28]. We suggest that entablature and scale changes in basalt could occur even without sudden changes in the external conditions. In starch, we observed transitions in scale using constant-power external conditions, in which the drying front is slowly, continuously, decelerating with depth. Starch columns are approximately 100 times smaller in diameter than basaltic columns. Directly scaling the $1 \mathrm{~cm}$ wide scale transitions in starch, we find a $1 \mathrm{~m}$ scale that corresponds reasonably well with the width of the entablatures observed in basalt. Such transitions could be the result of dynamical instabilities of the fracture pattern, which occur when a stable range of pattern scales is exceeded. An analogous instability occurs in directionally dried thin films, in which a periodic array of cracks can continue to propagate under a range of drying conditions [29]. Outside this range, the array becomes unstable, and makes a transition to a new spacing $[22,29]$.

Our dynamical instability-driven entablature hypothesis is testable by observing the striae widths in basaltic colonnades. Striae are "chisel" marks on the sides of columns, which record individual fracture advances as the cooling front advances [5]. By observing the variation of the striae width across a colonnade-colonnade scale transition, it should be possible to determine how the cooling conditions changed. A continuous evolution across such a boundary, rather than a matching discontinuity in striae scale, would confirm the absence of catastrophic changes in the external conditions.

Our experiments have focussed on exploring the 3D structure of columnar jointing in corn starch. We have directly observed the operation of a strong ordering process that results in the eventual saturation of the pattern statistics. This mature state of the pattern contains considerable disorder, which we suggest is due to the strongly nonequilibrium, complex dynamics of the joints. An alternate explanation of residual disorder is that the system's geometry evolves toward equilibrium but gets stuck in a local minimum of the free energy [23]. This scenario is difficult to completely rule out, but seems unlikely given the observed persistent mobility of the joints, which do not fluctuate about equilibrium positions. Several new dynamical models of columnar jointing have recently been proposed by Jagla [12,22], and Jagla and Rojo [23], some of which seem to capture, qualitatively, the disordered dynamics we find.

We have observed coarsening of the starch colonnade as it penetrates our samples. This coarsening proceeds through the termination of crack tips along column sides or joints. The coarsening can be halted in constant-rate experiments, revealing an important relationship between fracture advance rate and pattern scale. The observation of discontinuous transitions in scale, however, implies it is not a one-to-one relationship. Rather, as occurs with similar $2 \mathrm{D}$ patterns [29], the exact pattern scale will depend on the system's dynamical history, as well as on the current externally imposed conditions. This alone may significantly modify field interpretations of columnar jointing, as we have outlined above. However, the appreciation of quasihexagonal fracture as a non-equilibrium dynamical system can go much further, and a quantitative understanding of this pattern could provide volcanology, cryophysics, planetary physics, and pattern physics with novel diagnostic tools. These considerations motivate a continuing interest in this beautiful phenomenon. 
We thank M. Henkelman and the Mouse Imaging Centre for supplying access to microtomography equipment, and Zhenquan Lin, A. M. Jellinek, P.-Y. Robin, E. A. Jagla, and R. P. Young for helpful discussions.

\section{REFERENCES}

[1] Sir R. Bulkley S. R. S., Philos. Trans. R. Soc. London, 17 (1693) 708.

[2] O'Reilly J. P., Trans. R. Irish Acad., 26 (1879) 641.

[3] Huxley T. H., Physiography: An Introduction to the Study of Nature (MacMillan and Co., London) 1881, p. 204.

[4] French J. W., Trans. Geol. Soc. Glasgow, 17 (1922) 50.

[5] Degraff J. M. and Aydin A., Geol. Soc. Am., 99 (1987) 605.

[6] Müller G., J. Geophys. Res., 103 (1998) 15239.

[7] Müller G., J. Volcanol. Geotherm. Res., 86 (1998) 93.

[8] Müller G., J. Struct. Geol., 23 (2001) 45.

[9] Toramaru A. and Matsumoto T., J. Geophys. Res., 109 (2004) B02205.

[10] Seshadri K. V., J. Geol. Soc. India, 49 (1997) 452.

[11] Menger F. M., Zhang H., Caran K. L., Seredyuk V. A. and Apkarian R. P., J. Am. Chem. Soc., 124 (2002) 1140.

[12] Jagla E. A., Phys. Rev. E, 69 (2004) 056212.

[13] Budkewitsch P. and Robin P.-Y., J. Volcanol. Geotherm. Res., 59 (1993) 219.

[14] Peck D. L. and Minakami T., Geol. Soc. Am. Bull., 79 (1968) 1151.

[15] Aydin A. and DeGraff J. M., Science, 239 (1988) 471.

[16] Dufresne E. R., Corwin E. I., Greenblatt N. A., Ashmore J., Wang D. Y., Dinsmore A. D., Cheng J. X., Xie X. S., Hutchinson J. W. and Weitz D. A., Phys. Rev. Lett., 91 (2003) 224501.

[17] Jones E. M., (Apollo 15 Lunar Surface Journal; Hadley Rille; http://www.hq.nasa.gov/ office/pao/History/alsj/a15/a15.rille.html (1996)).

[18] Lachenbruch A. H., Geol. Soc. Am. Spec. Paper, 70 (1962) 69.

[19] Sletten R. S., Hallet B. and Fletcher R. C., J. Geophys. Res., 108 (2003) 8044.

[20] Mellon M. T., J. Geophys. Res., 102 (1997) 25617.

[21] Mallet R., Philos. Mag., 50 (1875) 122.

[22] Jagla E. A., Phys. Rev. E, 65 (2002) 046147.

[23] Jagla E. A. and Rojo A. G., Phys. Rev. E, 65 (2002) 026203.

[24] Hardee H. C., J. Volcanol. Geotherm. Res., 7 (1980) 211.

[25] Gray N. H., Anderson J. B., Devine J. D. and Kwasnik J. M., Math. Geol., 8 (1976) 617.

[26] Grossenbacher K. A. and McDuffie S. M., J. Volcanol. Geotherm. Res., 69 (1995) 95.

[27] Long P. E. and Wood B. J., Geol. Soc. Am. Bull., 97 (1986) 1144.

[28] Degraff J. M., Long P. E. and Aydin A., J. Volcanol. Geotherm. Res., 38 (1989) 309.

[29] Shorlin K. A., De Bruyn J. R., Graham M. and Morris S. W., Phys. Rev. E, 61 (2000) 6950. 